\title{
Impact of Electric Vehicle Routing with Stochastic Demand on Grid Operation
}

\author{
Oluwaseun Oladimeji, Alvaro Gonzalez-Castellanos, David Pozo, Skolkovo Institute of Science and Technology \\ Yury Dvorkin, Samrat Acharya, New York University
}

\begin{abstract}
Given the rise of electric vehicle (EV) adoption, supported by government policies and dropping technology prices, new challenges arise in the modeling and operation of electric transportation. In this paper, we present a model for solving the $\mathrm{EV}$ routing problem while accounting for real-life stochastic demand behavior. We present a mathematical formulation that minimizes travel time and energy costs of a EV fleet. The EV is represented by a battery energy consumption model. To adapt our formulation to real-life scenarios, customer pick-ups and drop-offs were modeled as stochastic parameters. A chance-constrained optimization model is proposed for addressing pick-ups and drop-offs uncertainties. Computational validation of the model is provided based on representative transportation scenarios. Results obtained showed a quick convergence of our model with verifiable solutions. Finally, the impact of electric vehicles charging is validated in Downtown Manhattan, New York by assessing the effect on the distribution grid.
\end{abstract}

Index Terms-Electric vehicle, Chance-constrained optimization, Vehicle routing problem

\section{NOMENCLATURE}

Indexes and Sets

$i, j, s \in J \quad$ Customer locations.

$i, j, s \in J_{0} \quad$ Transportation nodes, i.e., customers locations and depot; $\left|J_{0}\right|=|J|+1$.

$k \in K \quad$ Charging stations. $K=J_{0} \cap N$.

$n, m \in N \quad$ Nodes in the electric distribution network.

$(n, m) \in \mathcal{L}$ Power distribution lines.

$v \in V \quad$ Electric vehicles.

\section{Parameters}

$\epsilon_{j}$

$C_{v}$

$C^{\mathrm{E}}$

$C^{\mathrm{T}}$

$D_{i}$

$\underline{E}_{v} / \bar{E}_{v}$

$E T_{i}$

$\bar{L}_{v}$

$L T_{i}$

$M^{(\cdot)}$

$P_{i}$

$\underline{P}_{n} / \bar{P}_{n}$

$P_{n}^{\text {de }} / Q_{n}^{\text {de }}$

$\frac{Q}{R}_{n} / \bar{Q}_{n}$

$\frac{Q}{R} n$

$\underline{S}_{n} / \bar{S}_{n}$

$T_{i j}$

$\bar{T}_{k} / \underline{T}_{k}$

$\bar{T}_{v}^{\mathrm{rech}}$

$\underline{V} / \bar{V}$

Risk tolerance for demand satisfaction at node $j,[-]$. Vehicle power consumption rate,

$[\mathrm{kWh} / \mathrm{min}]$.

Cost of electricity,

$[\$ / \mathrm{kW}]$.

Unitary cost of time travelled,

[\$/min].

Amount of dropped off customers at $i$, [passengers]. Minimum/maximum battery charge level, [kWh]. Earliest time of arrival at $j$,

Vehicle capacity,

[min].

Latest time of arrival at $j$,

[passengers].

Large constant,

[min].

Amount of picked up customers at $i$,

Minimum/maximum nodal active demand, $[\mathrm{kW}]$

Local active/reactive demand at node $n$, [kW/kVar].

Minimum/maximum nodal reactive demand, [kVar].

Vehicle charging rate,

$[\mathrm{kWh} / \mathrm{min}]$.

Minimum/maximum nodal apparent demand, [kVA].

Travel time between nodes,

[min].

Minimum/maximum charging time,

Maximum allowed time before recharge, [min].

Minimum/maximum nodal voltage,

Variables

$\pi_{j}$

Variable used to prohibit sub-tours,

$\tau_{k v}$

Charging time spent by vehicle $v$,
[-].

[min].
This work was partially funded by the Skolkovo Institute of Science and Technology as a part of the Skoltech NGP Program (Skoltech-MIT joint project).

\begin{tabular}{|c|c|}
\hline $\begin{array}{l}e_{j v} \\
l_{v}^{0} \\
l_{j v}\end{array}$ & $\begin{array}{lr}\text { Energy level of vehicle } v \text { at node } j, & \text { [kWh]. } \\
\text { Vehicle load after leaving the depot, } & \text { [passengers]. } \\
\text { Vehicle load after visiting location } j, & \text { [passengers]. }\end{array}$ \\
\hline$p_{n m} / q_{n m}$ & $\begin{array}{l}\text { Active/reactive power flow along line }(n, m) \text {, } \\
{[\mathrm{kW} / \mathrm{kvar}] \text {. }}\end{array}$ \\
\hline$p_{n}^{\mathrm{d}}$ & $\begin{array}{l}\text { Sum of electric power consumption and EV charging } \\
\text { power at node } n, \\
{[\mathrm{~kW} / \mathrm{kvar}] .}\end{array}$ \\
\hline $\begin{array}{l}p_{n}^{\mathrm{g}} / q_{n}^{\mathrm{g}} \\
t_{j v}\end{array}$ & $\begin{array}{lr}\text { Active/reactive power generation at } n, & {[\mathrm{~kW} / \mathrm{kVar}] \text {. }} \\
\text { Arrival time of } v \text { to node } j, & \text { [min]. }\end{array}$ \\
\hline$u_{n}$ & Square of the voltage magnitude at $n$, \\
\hline$w_{k v}$ & $\begin{array}{l}\text { Auxiliary variable combining charge duration and } \\
\text { power usage at station, } \\
{[\mathrm{kWh}] .}\end{array}$ \\
\hline$x_{i j v}$ & $\begin{array}{l}\text { Binary variable indicating whether vehicle } v \text { trav } \\
\text { directly from } i \text { to } j,\end{array}$ \\
\hline$y_{k v}$ & $\begin{array}{l}\text { Binary variable indicating vehicle } v \text { at charging } \\
\text { station } k \text {, }\end{array}$ \\
\hline
\end{tabular}

Until recently, large scale electrification of the transportation sector has not gained so much attention, even though transportation represents more than $25 \%$ of the world's energy consumption [1]. The transportation sector contributes to a large share of greenhouse emissions, e.g., in California, it accounts for more than $40 \%$ of emissions [2]. Energy decarbonization road-maps highlight electrification of the transport sector as a critical step to reach emission goals. Likewise, emissions reductions are especially necessary for polluted mega-cities, where an increasing number of projects have replaced combustion-based bus fleets by electric ones. This modernization benefits from improvements in rechargeable battery technology leading to quantitative improvement in EV technology. EV manufacturing companies continue to make measurable production growth with a significant presence of EVs used for commercial transportation services [3].

In terms of impact of vehicles charging on the distribution grid, let us evaluate how much power is drawn from the grid during routing. Using a Tesla model $\mathrm{S}$ with a battery capacity of $75 \mathrm{kWh}$ with consumption of only about $33 \mathrm{kWh}$ for 100 miles driven translates to a driving range of about 220 miles. This might mean only a single charge in 2 days for private car owners which sit idle for about $95 \%$ of the time on a daily basis [4]. However, when we consider that ride sharing where cars would constantly be on the road, then the effect on the electric network are: more frequent charging sessions and increasing use of fast-charging stations.

If we consider fast chargers with power charging rate of $50 \mathrm{~kW}$, twenty vehicles would be withdrawing 1MW from the power network if they are charged simultaneously. New York uses about 11000MWh of electricity per day which translates into an average hourly consumption of approximately 450MW. Thus, only twenty vehicles would represent about $0.25 \%$ of the city's demand. An average ridesharing car makes between 5 to 12 trips per day. Thus, an EV would require charging at least once or twice per day. If we consider a fleet owner with $500 \mathrm{EVs}$, power requirement increases up to $25 \mathrm{MW}$ a considerable increase on demand is requested to the electric network.

Transportation electrification then calls for modification of previous vehicle routing algorithms, which have considered traditional internal 
combustion engine (ICE) vehicles. ICE vehicles estimate energy consumption by distance traveled and gross weight of the vehicle. For EVs, these assumptions are still valid, but additional considerations such as battery properties and charging infrastructure must also be considered.

\section{A. Literature Survey}

The vehicle routing problem (VRP) with simultaneous pickup and delivery for material goods was introduced in [5]. In [6], customer satisfaction was added as a model constraint. Time-window constraints are included in the VRP to prevent situations in which customers wait for long periods. A review on exact algorithms for time-and- capacityconstrained models was presented in [7]. Including uncertainty, [8] modelled the stochastic nature of drop-offs and pickups using chance constraints. In another study, non-linear interval-based programming was used to investigate VRP with uncertainty in drop-offs [9].

As energy management became increasingly important, various studies incorporated partial battery charging stations into their model while still using a conventional combustion engine model for energy management. The green-mixed fleet VRP was proposed in $|10|$. Models with minimization of traveled distance and energy usage have also been presented in [11]-[14].

Single user vehicles, due to their passive usage, are primarily considered as static energy storage equipment. However, in this paper we seek to address the question on EVs applicability based on two premises: 1) EVs production continues to increase, and 2) ride/car sharing popularity, which increases vehicle mileage. As EVs increase in numbers, there will be an increasing request for power reliability from the existing power infrastructure. Additionally, if EVs are widely accepted as an alternative to commercial vehicles, we pose a further question of how much impact this would have on the power grid.

The current solution being proposed to curtail the impact of EVs on the power grid is valley filling. Inherent challenges with this is the possibility of creating a new peak and importantly, controlling human behaviour. Valley filling [15] essentially seeks to smooth out the individual or aggregate load curve by shaving peak loads and increasing off-peak loads e.g. charging EVs at night.

In [16], [17], offline and online valley filling algorithms were introduced. The authors recognised the challenge posed by large fleet of EVs on the distribution network if the EVs are stationed in residential areas. The EV charging problem was modelled using a modified version of the optimal power flow problem with a valley-filling objective. However, electricity price was considered constant in the papers and EVs were taken as stationary. A mode of decentralized coordination of charging large populations of EVs using non-cooperative game theory was developed in [18]. The charging schedule was also modeled as a valley-filling problem. As in the previous literature discussed, electricity price was taken as constant and uncertainties were not considered. A control algorithm focusing on peak shaving was proposed to match the power curve and scheduled EV usage curve in [19]. A grid that incorporates smart meters, intelligent switches, two-way charge devices and a central control center was considered. The charging decision is then made by matching the load forecast curve and the available charge/discharge curve of EVs.

A major drawback in these systems is the absence of mobilityawareness when scheduling EV charging. An additional challenge was lack of real data in justifying the proposed control algorithms/frameworks developed. This is also a reason why authors modeled electricity prices as time-invariant due to lack of evidence regarding how the EVs will affect the dynamics of the power system.
Furthermore, several tools have been developed to address some of the questions that we pose in our work. CASPOC, COMPOSE and HOMER are three simulators that are closest to our proposed methodology [20]. However, these tools have limitations which do not capture the solution that we propose. HOMER, for instance, does not include mobility and time dependent batteries based on specific EVs could not be modeled on it. CASPOC and COMPOSE, though can model EVs, do not support integration of mobility with the grid.

This paper introduces a new variant of co-optimization of multienergy systems which have included coordinated operation of power, thermal and gas networks. An operational optimization model for electricity, thermal and gas network was presented in [21]. Coordinated operation of the heating and electricity system for the United Kingdom is discussed in [22]. A survey on power and gas system integration is presented [23]. These coordinated systems offer environmental, system reliability, and economic benefits; similar benefits can be achieved by the coordination of the transportation and electricity network.

\section{B. Paper Contribution and Organization}

The main contribution of this work is to propose a co-optimization model that combines the routing of an EV transportation fleet and the distribution grid power flow. Additionally, we extend the proposed model by incorporating pick-up and drop-off uncertainty in the VRP model. The resulting model is formulated as a chance-constrained optimization problem and reformulated as an equivalent deterministic mixed-integer linear programming (MILP). Then, we present an illustrative case study based on real data of the island of Manhattan, New York.

The rest of the paper is organized as follows. Section [II develops the mathematical formulation of the VRP problem with vehicle recharging, demand uncertainty and power flow in the electric distribution network. In Section III] we describe the test case data, and how it is processed and used for computational tests. In Section IV the proposed model is tested on the case study. Section $\mathrm{V}$ concludes the study.

\section{Mathematical Model}

The overall aim of the optimization problem is to reduce the EV operation cost by minimizing travel time and energy usage of the vehicles and to minimize the cost of the distribution network operation by efficiently scheduling EV charging periods. This section is devoted to formulating electric transport routing, representative energy management model and the distribution grid. Noting that some parameters are not exactly known during planning, equations used to describe stochasticity will also be highlighted. Our goal is to provide a routing and EV charging plan for EV-fleet owners and electric utility companies. This plan will enable a reduction in distance traveled, contribute to reduced energy usage and consequently lead to efficient and operation of the distribution grid.

\section{A. Electric Vehicle Routing Model}

The vehicle routing problem consists of assigning routes to a fleet of transportation vehicles based on drop-off and pick-up needs of customers distributed on a set of nodes on the transportation network. The objective [1a) of the VRP is the minimization of total time traveled by the transportation fleet. $x_{i j v}$ represents whether the vehicle $v$ travels directly from stop $i$ to stop $j$, and $T_{i j}$ is the distance between these stops. In combination with (1a), the VRP can 
be modelled by the following problem.

$$
\begin{aligned}
& \min \sum_{i \in J_{0}} \sum_{j \in J_{0}} \sum_{v \in V} x_{i j v} T_{i j} \\
& \text { s.t.: } \sum_{i \in J_{0}} \sum_{v \in V} x_{i j v}=1 \text {, } \\
& \sum_{i \in J_{0}} x_{i s v}=\sum_{j \in J_{0}} x_{s j v}, \\
& l_{v}^{0}=\sum_{i \in J_{0}} \sum_{j \in J} D_{j} x_{i j v}, \\
& l_{j v} \geq l_{i v}-D_{j}+P_{j}-M_{i j v}^{L}, \\
& l_{j v} \leq \bar{L}_{v}, \\
& \pi_{j} \geq \pi_{i}+1-\left|J_{0}\right|\left(1-\sum_{v \in V} x_{i j v}\right), \\
& E T_{j} \leq t_{j v} \leq L T_{j}, \\
& t_{j v} \geq t_{i v}+\tau_{k v}+x_{i j v} T_{i j}-M_{i j v}^{T} \\
& l_{j v}, t_{j v}, \pi_{j} \geq 0 \text {, } \\
& x_{i j v} \in\{0,1\},
\end{aligned}
$$

Constraints $1 \mathrm{~b}$ and $(1 \mathrm{c})$ define that each customer should be moved from a pick-up point to a drop-off point by exactly one vehicle. Initial vehicle load from the depot is set by (1d). 1e represents the vehicle loads en route; with $M_{i j v}^{L}=M^{L}\left(1-x_{i j v}\right)$. The constant $M^{L}$ is a large number that sets a common lower bound to constraints in which $x_{i j v}$ has a unitary value. The value of $M^{L}$ can be calculated beforehand based on demand levels of the transportation network. Vehicle capacity limits are set by [1f].

As the algorithm searches for solutions to satisfy given constraints, many disjointed routes which have no connections with the depot will be created. However, a fleet manager requires all vehicles to be returned to the starting station at the end of every tour. Sub-tour eliminating constraint, $1 \mathrm{~g}$, , removes solutions without connections to the depot.

Based on customer-established pick-up times, expression (1h) sets the bounds for the arrival time at different stops. Arrival time is calculated with [1i), where the value $M_{i j v}^{T}$ is calculated analogously as $M_{i j v}^{L}$, but with a large time constant $M^{T}$. The lower bounds on vehicle load, traveled time and sub-tour breaking variable is set by [1j), whereas $\left[1 \mathrm{k}\right.$ defines $x_{i j v}$ as a binary variable.

\section{B. Energy Usage Model}

For the routing of the electric fleet, it is necessary to model energy usage of the vehicle and time spent at charging stations. The objective of the VRP with energy management can be set either as the minimization of the total time spent at charging stations or as the total cost of energy used. If a constant electricity price is considered, both objectives are equivalent. Thus, we express the objective of the VRP with energy management as:

$$
\begin{aligned}
& \min \sum_{k \in K} \sum_{v \in V} \tau_{k v} \\
& \text { s.t.: } e_{j v} \leq e_{i v}+\tau_{k v} R_{v}-C_{v} x_{i j v} T_{i j}+M_{i j v}^{E}, \quad \forall i \neq j, k, v \\
& \sum_{v \in V} x_{i j v} T_{i j} \leq \bar{T}^{\mathrm{rech}}, \quad \forall i, j \\
& \underline{T}_{k} y_{k v} \leq \tau_{k v} \leq y_{k v} \bar{T}_{k}, \quad \forall k, v \\
& e_{k v}+\tau_{k v} R_{v} \leq \bar{E}_{v}, \quad \forall k, v \\
& \underline{E}_{v} \leq e_{j v} \leq \bar{E}_{v} \\
& \forall j, v \text {. }
\end{aligned}
$$

Battery energy levels at each node are calculated by $2 \mathrm{~b} . M_{i j v}^{E}=$ $M^{E}\left(1-x_{i j v}\right)$ is a large value that is used to activate this constraint (2b) when the edge connecting the stops $i$ and $j$ is traveled. Expression 2c sets an upper bound on the time a vehicle can move before returning to depot. (2d) and 2e respectively define the lower and upper bounds on the time of charging for a vehicle visiting a charging station and the energy after a charging stop. Battery capacity limits are defined by (2f). Battery degradation is not considered in our formulation due to modern battery technology which are designed to ensure a good lifetime. Studies have shown that EV battery degradation rate is non-linearly correlated with its usage, and for EV fleets, increased utilization does not necessarily impact battery life [24].

\section{Distribution Grid Model}

The conventional methods for solving power flow in distribution grid follow the fundamental electrical current flow laws. We formulate the electrical distribution grid using LinDistFlow equations [25] which is a linear form of the power flow equations in electrical distribution grids, resulting into a computationally and numerically efficient formulation for distribution network operation modeling.

$$
\begin{array}{lll}
p_{n m}=p_{m}^{\mathrm{g}}-P_{m}^{\mathrm{de}}-\sum_{m^{\prime}:\left(m, m^{\prime}\right) \in \mathcal{L}} p_{m m^{\prime}}, & & \forall(n, m) \in \mathcal{L} \\
q_{n m}=q_{m}^{\mathrm{g}}-Q_{m}^{\mathrm{de}}-\sum_{m^{\prime}:\left(m, m^{\prime}\right) \in \mathcal{L}} q_{m m^{\prime}}, & & \forall(n, m) \in \mathcal{L} \\
u_{m}=u_{n}-2\left(r_{n m} p_{n m}+x_{n m} q_{n m}\right), & & \forall(n, m) \in \mathcal{L} \\
p_{n m} \leq \bar{P}_{n m}, & & \forall(n, m) \in \mathcal{L} \\
q_{m n} \leq \bar{Q}_{n m}, & & \forall n, m) \in \mathcal{L} \\
\underline{P}_{n} \leq p_{n}^{\mathrm{g}} \leq \bar{P}_{n}, & \forall n \\
\underline{Q}_{n} \leq q_{n}^{\mathrm{g}} \leq \bar{Q}_{n}, & \forall n, \\
\underline{V}_{n}^{2} \leq u_{n} \leq \bar{V}_{n}^{2}, & \forall n
\end{array}
$$

Where $P_{n}^{\mathrm{de}}+i Q_{n}^{\mathrm{de}}$ represents the complex power consumption of electric demand only at node $n . p_{n m}+i q_{n m}$ is complex power flowing along the power line $(n m) \cdot p_{n}^{\mathrm{g}}+i q_{n}^{\mathrm{g}}$ is the power generation at node n. $u_{n}$ is the square of the nodal voltage. Equations 3a and $3 \mathrm{~b}$ model power flow through the lines. The nodal voltage is calculated by (3c); while line flow, generation, and voltage limits are set by $3 \mathrm{~d}-3 \mathrm{~h}$.

\section{Transportation Network and Electrical Grid Coupling}

The transportation network and the electric grid are coupled by EV charging stations. The power required by EVs during charging should be added to the grid's existing demand. Note that the location and power demand from EVs are dependent on the routes resultant of the VRP. Electricity consumption in the distribution grid can be summed up to that in the transportation grid at the nodes where charging stations are present by the following expression:

$$
p_{n}^{\mathrm{d}}=p^{\mathrm{d}, e}+R_{v}, \quad(\forall n \in J, \forall k \in K) \wedge y_{k v}=1 .
$$

Thus, active power consumption $p_{n}^{\mathrm{d}}$ at node $n$, is equivalent to regular electrical load $p^{\mathrm{d}, e}$, plus electric load related with transportation grid, $R_{v}$, at charging station $k$.

\section{E. Modelling Uncertainty}

Given the uncertain nature of demand, we need to express the uncertainty in the drop-off and pick-up parameters of the transportation demand balance (1e). We opt for the use of chance-constrained programming to describe demand uncertainty because this approach allows us to satisfy customer demands with a certain degree of confidence, i.e., we model the constraints in such a way that drop-offs and pick-ups are complied with a probability above the established level $\left(1-\epsilon_{j}\right)$ :

$$
\mathbb{P}\left(l_{j v} \geq l_{i v}-D_{j}+P_{j}-M_{i j v}^{L}\right) \geq 1-\epsilon_{j}, \quad \forall i \neq j, v
$$


where $l_{i v}$ and $l_{j v}$ are variables representing vehicle nodal loads, and $D_{j}$ and $P_{j}$ are bounded parameters. The probability of the nodal constraint satisfaction is given by $\left(1-\epsilon_{j}\right) \in[0,1]$. The use of nodal constraint satisfaction allows us to assign differential priority to transportation nodes based on their importance by selecting different values of the risk parameter $\epsilon_{j}$, i.e., more important transportation nodes have lower risk values $\epsilon_{j}$. The following reformulation of vehicle capacity conservation is made |26]:

$$
l_{j v} \geq l_{i v}-\mathbb{E}\left[D_{j}-P_{j}\right] \pm \Phi_{1-\epsilon_{j}}^{-1} \sigma_{j}-M_{i j v}^{L}, \quad \forall i \neq j, v
$$

The value $\mathbb{E}\left[D_{j}-P_{j}\right]$ represents the net demand expected at node $j$. $\Phi_{1-\epsilon_{j}}^{-1}$ is the inverse cumulative distribution function evaluated at $1-\epsilon_{j}$, and $\sigma_{j}$ the standard deviation of the nodal net demand.

\section{F. Overall Objective}

We consider writing a single objective by making some adjustments to our prior objectives defined above.

- A penalization factor $C^{\mathrm{T}}$ is introduced to assign a cost related to traveling time in 1a. This factor is similar to a transport service cost based on distance or time traveled.

- By considering power consumption and time spent charging at different nodes, multiplied by electricity cost, it is possible to obtain the total cost of energy. The electricity cost is constant here because of the resolution of our planning horizon which is within an hour or less and the electricity cost is generally nonvariant within this time period. Thus, the electricity cost will be updated according to prevalent prices during the time when the planning schedule is required.

The new objective function becomes:

$$
\min \sum_{i, j, v} C^{\mathrm{T}} x_{i j v} T_{i j}+\sum_{v, k} C^{\mathrm{E}} p_{k}^{c} \tau_{k v}
$$

The second part of the final objective (7) introduces non-convexity to the model because both power consumption and charging time are variables. However, power consumption and charging time are both bounded by battery capacity and charge rate. We overcome the non-convexity by combining these two variables via the introduction of a McCormick envelope [27] which is represented by the set of constraints 8 :

$$
\begin{array}{ll}
w_{k v} \leq \bar{E}_{v} \tau_{k v}+p_{k}^{c} \underline{T}_{k}-\bar{E}_{v} \underline{T}_{k}, & \forall k, v \\
w_{k v} \leq p_{k}^{c} \bar{T}_{k}+\underline{E}_{v} \tau_{k v}-\underline{E}_{v} \bar{T}_{k}, & \forall k, v \\
\underline{E}_{v} \leq p_{k}^{c} \leq \bar{E}_{v}, & \\
\underline{T}_{k} \leq \tau_{k v} \leq \bar{T}_{k}, & \forall k, v
\end{array}
$$

A linear representation of the objective is finally given by:

$$
\min \sum_{i, j, v} C^{\mathrm{T}} x_{i j v} T_{i j}+\sum_{v, k} C^{\mathrm{E}} w_{k v}
$$

\section{G. Model Summary}

For further analysis, we classify our formulation into two broad groups: deterministic and chance-constrained stochastic models. As shown in Table I] the main difference in formulation appears in the set of constraints defining the EV routing problem.

\section{A. Transportation Network Data}

$$
\text { III. CASE STUdY }
$$

\begin{tabular}{|c|c|c|}
\hline & Deterministic & Stochastic CC \\
\hline Objective & & 9 ) \\
\hline EV routing problem & $1 \mathrm{~b}-1 \mathrm{k}$ & (1b) $-11 d$ \\
\hline Energy usage & $2 b-2 f$ & $2 b-\sqrt{2 f}$ \\
\hline Electric distribution $\mathrm{OPF}$ & (3) & (3) \\
\hline Coupling constraint & 4 & (4) \\
\hline Total charged energy & 8 & 8 \\
\hline
\end{tabular}

The case study selected for validating our proposed methodology is based on the Downtown Manhattan transportation network. Dropoff and pick-up data is taken from taxi trip records by the New York City Taxi and Limousine Commission for 2018 [28]. The data set includes pick-up and drop-off dates, times, locations, trip distances and driver-reported passenger counts. Table II shows a snippet from this data set where PULID and DOLID are respectively the pick-up
TABLE I: Model Summary

TABLE II: Representative Data Snippet

\begin{tabular}{cccccr}
\hline $\begin{array}{c}\text { Pickup } \\
\text { date-time }\end{array}$ & $\begin{array}{c}\text { Drop-off } \\
\text { date-time }\end{array}$ & $\begin{array}{c}\text { Trip } \\
\text { distance }\end{array}$ & PULID & DOLID & $\begin{array}{c}\text { Passenger } \\
\text { count }\end{array}$ \\
\hline \hline $12 / 31 / 2018$ & $\begin{array}{c}1 / 1 / 2019 \\
12: 37: 04\end{array}$ & 16.6 & 162 & 26 & 1 \\
$11: 58: 45$ & & & & 170 & 2 \\
\hline $12 / 31 / 2018$ & $1 / 1 / 2019$ & 3 & 144 & & 1 \\
$11: 58: 44$ & $12: 16: 54$ & & & & \\
\hline $12 / 31 / 2018$ & $1 / 1 / 2019$ & 6.4 & 162 & 13 & \\
$11: 58: 37$ & $12: 14: 04$ & & & & \\
\hline
\end{tabular}

location ID and drop-off Location IDs, which indicate exact latitudes and longitudes of these locations.

In order to use the data in our proposed VRP model, it was necessary to process through clusterization, while preserving its accuracy. The first opportunity for aggregation comes from passenger count. Taxis routinely pick up just one passenger per trip. Here, we extend our focus beyond one passenger scenario to ride-sharing cases. With ride-sharing, passengers can be added to each vehicle in the fleet until it reaches its capacity. This aggregation makes it possible to add all demand for an extended period, for example all drop-offs and pickups between $6 \mathrm{am}$ and $11 \mathrm{am}$ are aggregated and the case is evaluated such that all the demands are within a 2-hour interval.

Using latitudes and longitudes, we divided the data into distinct locations such as commercial, industrial, and residential regions. With this, we were able to track movement patterns at different times of the day. For example, people generally move from residential areas to commercial centers in the mornings and vice versa in the evenings. The routes from the solution of the model ensures that vehicles can pick up passengers until their capacity is exhausted while still fulfilling the pick-up time and other constraints.

Fig. 1a shows a geographical snapshot of the demand data, which is at the Downtown Manhattan grid, where green squares represent originating nodes, red circles represent destination nodes and large blue triangles are EV charging stations.

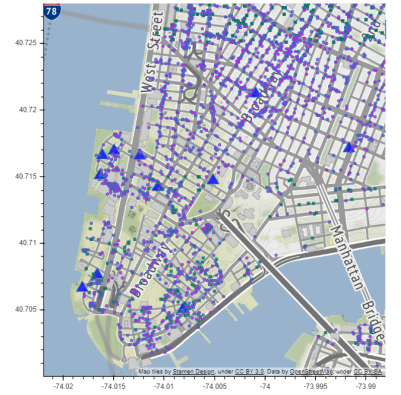

(a) Passenger concentration and EV CS in Downtown Manhattan btw 6-10am, 13 Aug '18

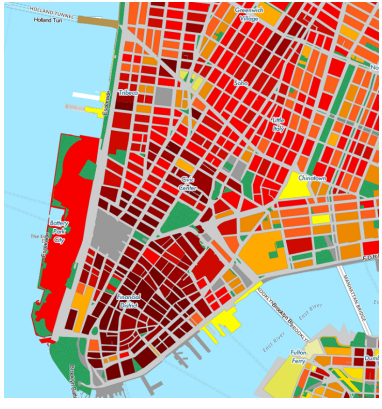

(b) Downtown Manhattan energy consumption by area
Fig. 1: Downtown Manhattan transport and energy demands 


\section{B. Electric Network Data}

For local electricity consumption, we obtained information from [29] and New York City Mayor's Office of the Long-Term Planning and Sustainability [30]. Electricity consumption at each block and lot level was obtained from [29] and consumption patterns are shown by the different shades of red in Fig. 1b Darker red and lighter/yellow colors show regions of high and low power consumption respectively. Voltage ratings of each charging station location and reactance between stations were estimated similarly to [31].

\section{Computational tests}

We compare the results of the deterministic and stochastic optimization here using the cases defined in Section II-G For the deterministic model, known input include data outlined in Table II and power requirements while variables to be determined include the route and energy consumption of each vehicle. For the stochastic model, demands at each node is unknown and modeled after a Gaussian distribution following analysis of historical data.

For clarity, we chose a 15-location case from the original data set with about 600 passengers over a 2-hour period. We consider a modest number of 6 vehicles deployed by the fleet owner to meet these demands, resulting in a model with over 1350 variables. As number of locations and vehicles increases, so does the dimension of $x_{i j v}$. Since there are $36 \mathrm{EV}$ charging stations in Downtown Manhattan, (Fig. 1a), distance mapping is used to determine the nearest charging station when vehicles run out of charge or when power available would not be enough to reach the next passenger location.

\section{A. Routing Results}

Using historical data, Fig. 2a shows the routing results under assumption of perfect demand knowledge, i.e., deterministic modeling. Only 5 out of 6 available vehicles were deployed from the depot. However, when uncertainty is introduced in this scenario, result of routes varies from the deterministic model. It is shown in Fig. 2b that the routing is changed and also an additional vehicle is deployed. This is the case when a high degree of confidence is used, i.e., $1-\epsilon_{j} \geq 99.5 \%$.

However, for $90 \%<1-\epsilon_{j}<95 \%, 5$ vehicles were deployed, but with a different set of routes. The confidence levels translate to how many passengers will be picked or dropped in a particular location. Thus, as the tolerated risk varies, the optimal amount of vehicles and routes also change.

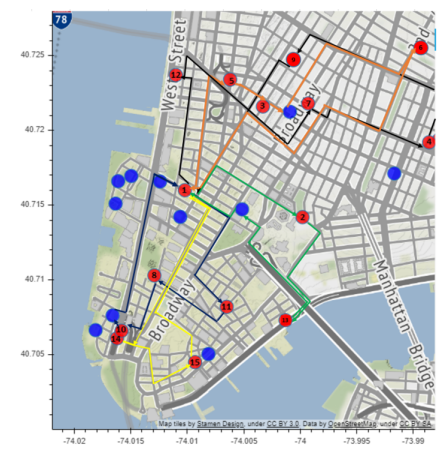

(a) Routing plan for deterministic model

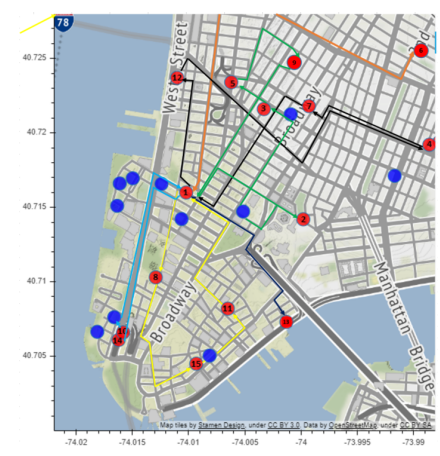

(b) Routing plan for chanceconstrained model
Fig. 2: Results of vehicle routing problem

Table III presents the optimization results of different test cases. Labels starting with 'D' and 'U' represent deterministic and stochastic problems respectively. Here, we contrast results of deterministic versus stochastic chance-constrained model. For few locations,
$|J| \leq 25$, number of routes and objective value have similar value for both types of models. However, as number of locations $|J|$ increases, number of routes differ greatly between both models. The same trend is seen with the objective value. The number of routes is equivalent to number of vehicles deployed from the fleet while the objective function, 9, represents cost of travelling plus cost of energy usage for the deployed EVs. The increase in number of vehicles deployed can be thought of as the price paid to satisfy an uncertain demand.

TABLE III: Results Summary

\begin{tabular}{lrrrr}
\hline \multicolumn{1}{c}{ Code } & Locations & Expected Passengers & Routes & Objective [\$] \\
\hline \hline D-L5 & 5 & 224 & 2 & 1.32 \\
U-L5 & 5 & 224 & 2 & 1.32 \\
D-L10 & 10 & 492 & 4 & 0.87 \\
U-L10 & 10 & 492 & 6 & 1.51 \\
D-L15 & 15 & 696 & 5 & 1.12 \\
U-L15 & 15 & 696 & 6 & 1.14 \\
D-L20 & 20 & 904 & 8 & 1.33 \\
U-L20 & 20 & 904 & 9 & 1.33 \\
D-L25 & 25 & 1196 & 11 & 1.10 \\
U-L25 & 25 & 1396 & 12 & 1.12 \\
D-L30 & 30 & 1396 & 12 & 1.31 \\
U-L30 & 30 & 2144 & 18 & 1.31 \\
D-L50 & 50 & 2144 & 20 & 1.43 \\
U-L50 & 50 & 3208 & 35 & 2.43 \\
D-L75 & 75 & 3208 & 38 & 2.12 \\
U-L75 & 75 & 4424 & 65 & 2.66 \\
D-L100 & 100 & 4424 & 72 & 2.50 \\
U-L100 & 100 & & & 3.22 \\
\hline
\end{tabular}

\section{B. Energy Consumption}

Fig. 3 presents the battery energy levels at different locations for scheduled routes for the 15-node case. The locations visited by each vehicle are represented in the spider web diagram while the height of spike is battery level of the EV. Where the line representing each vehicle changes direction from downward-movement to upwardmovement depicts a charging station. In this 15-node problem, the charging stations are located at nodes 1, 2, 6, 8, 9 and 10. Take Vehicle 1 in Fig. 3a for example. Its route plan is 1-11-8-10-1 and this plan takes it through two charging stations at nodes 8 and 10 . However, it only charges at one of them, node 8 . The route is complete when it returns to the depot and again recharges to the full energy level.

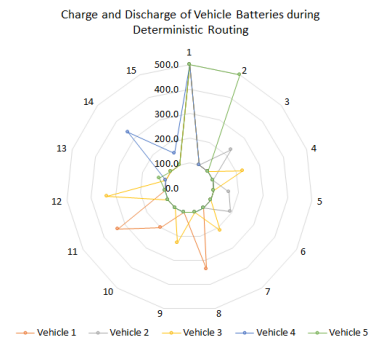

(a) Energy levels resulting from Deterministic model

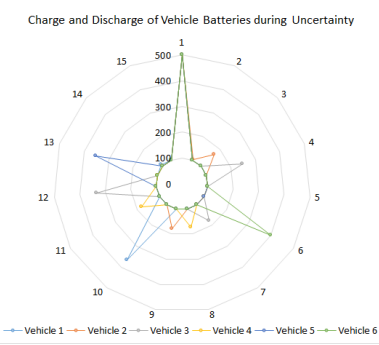

(b) Energy levels resulting from chance-constrained model
Fig. 3: Energy levels during routing

\section{Energy Consumption and $\mathrm{CO}_{2}$ e Emissions}

In this section we analyse possible emissions' reductions when EVs are deployed instead of ICE vehicles for city-wide transportation. $5 \%$ of power required by public transit in NYC is from electricity while $80 \%$ comes from petroleum. For the types of vehicles listed, the emission factor is $2.26 \mathrm{KgCO}_{2} \mathrm{e} /$ unit of fuel and approximately 3.4 million gallons of gasoline and diesel fuel are used per day 
[30|. The average GHG emissions then stands at $19.23 \mathrm{kgCO}_{2}$ e per vehicle. On the other hand, the emission factor for electricity is $0.257 \mathrm{kgCO}_{2} \mathrm{e} / \mathrm{kWh}$.

Tables IV and $\mathrm{V}$ present the energy consumption and emissions results for the deterministic and the stochastic chance-constrained cases respectively. The value of GHG emissions for liquid fuel vehicles were estimated using the fuel emission factors above and a fuel-oil equivalent of energy used by EVs. Approximately, a $43 \%$ reduction would be made in GHG emissions if EVs are utilized for shared taxi, as seen in Table IV When shared vans are used, which can accommodate between 8 and 10 passengers, we observe even greater savings. Van utilization lowers the number of vehicles, leading to a reduction in total $\mathrm{CO}_{2} e$ emissions. If the energy consumption by vans and taxis is scaled based on number of passengers, emission by vans would be about $10 \%$ of that of taxis.

TABLE IV: Total Emissions for Deterministic Demand

\begin{tabular}{lrrrr}
\hline \multicolumn{1}{c}{ Type } & Vehicles & Passengers & $\begin{array}{c}\text { Energy use } \\
{[\mathrm{kWh}]}\end{array}$ & $\begin{array}{r}\text { Emissions } \\
{\left[\mathrm{kgCO}_{2} e\right]}\end{array}$ \\
\hline \hline Taxi (EV) & 12 & 30 & 402.9 & 103.5 \\
Van (EV) & 5 & 150 & 228 & 58.6 \\
Taxi (Liquid fuel) & 12 & 30 & - & 815.4 \\
Van (Liquid fuel) & 5 & 150 & - & 461.04 \\
\hline
\end{tabular}

TABLE V: Total Emissions with Stochastic Demand

\begin{tabular}{lrrrr}
\hline Type & Vehicles & $\begin{array}{c}\text { Expected } \\
\text { passengers }\end{array}$ & $\begin{array}{c}\text { Energy use } \\
{[\mathrm{kWh}]}\end{array}$ & $\begin{array}{r}\text { Emissions } \\
{\left[\mathrm{kgCO}_{2} e\right]}\end{array}$ \\
\hline \hline Taxi (EV) & 14 & 30 & 422.9 & 108.7 \\
Van (EV) & 6 & 150 & 248.9 & 64.0 \\
Taxi (Liquid fuel) & 14 & 30 & - & 854.3 \\
Van (Liquid fuel) & 6 & 150 & - & 503.75 \\
\hline
\end{tabular}

\section{Conclusion}

This paper describes a new mixed integer linear programming formulation for EV routing which incorporates demand uncertainty, EV energy modelling and electric power grid utilization. Formulations were done such that bounds on confidence levels, simplicity and true representation of the systems we intended to describe were preserved. To reflect real life scenarios, we proposed a chance-constrained formulation for uncertainty. We thereafter based our case studies on inferences from real transport and electric network data of New York City. For our computational tests, differences between deterministic and stochastic model solutions were presented and analysed. This was done using the analysis of routing, energy consumption and emission of EVs deployed from the fleet.

Results from deterministic and stochastic models were contrasted for large number of locations and we noted that the increase in objective value for stochastic model is the extra cost of uncertainty. This increase in the objective value is reflected both in the amount of deployed vehicles and in their total energy consumption. Finally, we exhibit equivalent $\mathrm{CO}_{2}$ emissions' reduction when EVs are used as taxis and as shared vehicles/vans. We show that there is a significant emissions' reduction when EVs are utilized for commercial transportation and we opine that this is a viable solution for climate change mitigation. The effect of a single aggregator of EVs on emissions reduction has been shown here. Future research direction could be investigating the cost/benefit for individual fleet owners if they collaborate with other fleet owners. Other advantages which include power system support and provision of ancillary services could be realized.

\section{REFERENCES}

[1] U. S. Energy Information Administration, "Energy Consumption By Sector," https://www.eia.gov/totalenergy/data/monthly/, 2019.
[2] California Air Resources Board, "California Greenhouse Gas Emissions for 2000 to 2017," 2019.

[3] IEA (2019), "Global EV Outlook 2019," 2019, iEA, Paris.

[4] P. Barter, "Cars are parked 95\% of the time," 2016.

[5] H. Min, "The multiple vehicle routing problem with simultaneous delivery and pick-up points," Transportation Research Part A: General, vol. 23, no. 5, pp. 377-386, 1989.

[6] J. Dethloff, "Vehicle routing and reverse logistics," OR-Spektrum, vol. 23, no. 1, pp. 79-96, 2001.

[7] R. Baldacci, A. Mingozzi, and R. Roberti, "Recent exact algorithms for solving the vehicle routing problem under capacity and time window constraints," Eur. J. Op. Res., vol. 218, no. 1, pp. 1-6, 2012.

[8] N. Mahdi and B. Chen, "Vehicle routing with probabilistic capacity constraints," Eur. J. Op. Res., vol. 270, pp. 544-555, 2018.

[9] E. Cao, R. Gao, and M. Lai, "Research on the vehicle routing problem with interval demands," Ap. Math. Mod., vol. 54, pp. 332-346, 2018.

[10] G. Macrina et al., "The green mixed fleet vehicle routing problem with partial battery recharging and time windows," Comp. \& Op. Res., vol. 101, pp. 183-199, 2019.

[11] G. Hiermann et al, "The electric fleet size and mix vehicle routing problem with time windows and recharging stations," Eur. J. Op. Res., vol. 252, no. 3, pp. 995-1018, 2016.

[12] M. Schneider, A. Stenger, and J. Hof, "An adaptive vns algorithm for vehicle routing problems with intermediate stops," OR Spectrum, vol. 37, no. 2, pp. 353-387, 2015.

[13] S. Zhang et al, "Electric vehicle routing problem with recharging stations for minimizing energy consumption," Int. J. of Prod. Econ., vol. 203, pp. 404-413, 2018

[14] G. Ferro, M. Paolucci, and M. Robba, "An optimization model for electrical vehicles routing with time of use energy pricing and partial recharging," IFAC-PapersOnLine, vol. 51, no. 9, pp. 212-217, 2018.

[15] L. Jian, Y. Zheng, and Z. Shao, "High efficient valley-filling strategy for centralized coordinated charging of large-scale electric vehicles," Applied Energy, vol. 186, pp. 46-55, 2017.

[16] N. Chen et al, "Optimal charging of electric vehicles in smart grid: Characterization and valley-filling algorithms," pp. 13-18, 2012.

[17] N. Chen, C. W. Tan, and T. Q. S. Quek, "Electric vehicle charging in smart grid: Optimality and valley-filling algorithms," IEEE Journal of Selected Topics in Signal Processing, vol. 8, no. 6, pp. 1073-1083, 2014.

[18] Z. Ma, D. S. Callaway, and I. A. Hiskens, "Decentralized charging control of large populations of plug-in electric vehicles," IEEE Transactions on Control Systems Technology, vol. 21, no. 1, pp. 67-78, 2013.

[19] Z. Wang and S. Wang, "Grid power peak shaving and valley filling using vehicle-to-grid systems," IEEE Trans on PD, vol. 28, no. 3, pp. 1822-1829, 2013.

[20] K. Mahmud and G. E. Town, "A review of computer tools for modeling electric vehicle energy requirements and their impact on power distribution networks," Applied Energy, vol. 172, pp. 337-359, 2016.

[21] Wang, Yongli et al, "Operation optimization of regional integrated energy system based on the modeling of electricity-thermal-natural gas network," Applied Energy, vol. 251, p. 113410, 2019.

[22] Zhang, Xi et al, "Economic assessment of alternative heat decarbonisation strategies through coordinated operation with electricity system-uk case study," Applied Energy, vol. 222, pp. 79-91, 2018.

[23] M. Farrokhifar, Y. Nie, and D. Pozo, "Energy systems planning: A survey on models for integrated power and natural gas networks coordination," Applied Energy, vol. 262, p. 114567, 2020.

[24] GEOTAB, "What can 6,000 electric vehicles tell us about ev battery health?" 2019.

[25] M. Baran and F. F. Wu, "Optimal sizing of capacitors placed on a radial distribution system," IEEE Transactions on power Delivery, vol. 4, no. 1, pp. 735-743, 1989.

[26] M. Lubin, Y. Dvorkin, and L. Roald, "Chance constraints for improving the security of ac optimal power flow," IEEE Transactions on Power Systems, vol. 34, no. 3, pp. 1908-1917, 2019.

[27] J. Dombrowski, "Mccormick envelopes," 2015.

[28] New York (N.Y.). Taxi and Limousine Commission, "New York City Taxi Trip Data, 2009-2018. Ann Arbor,” 2019, feb 2019.

[29] B. Howard, L. Parshall, J. Thompson, S. Hammer, J. Dickinson, and V. Modi, "Spatial distribution of urban building energy consumption by end use," Energy and Buildings, vol. 45, pp. 141-151, 2012.

[30] PlanNYC, "A stronger, more resilient new york," Mayor's Office, New York, vol. 1, no. 1, pp. 1-223, 2013.

[31] S. Acharya, Y. Dvorkin, and R. Karri, "Public plug-in electric vehicles+ grid data: Is a new cyberattack vector viable?" IEEE Trans on $S G$, vol. 11, no. 6, pp. 5099-5113, 2020. 